Pacific Journal of Mathematics

ONE-DIMENSIONAL PERTURBATIONS OF OPERATORS 


\title{
ONE-DIMENSIONAL PERTURBATIONS OF OPERATORS
}

\author{
JOSEPH G. STAMPFLI
}

The purpose of this note is three-fold. First, to show that certain rank one operators of small norm will split off a unitary piece from the shift; second, to apply this technique to the "Berg construction"; and, third, to exhibit a normal operator whose eigenspace structure is drastically altered by the addition of a rank one operator.

Throughout this paper $\mathscr{L}(\mathscr{H})$ will denote the algebra of bounded linear operators on a separable Hilbert space $\mathscr{H}$.

1. We begin with a proposition which tells us which rank one operators preserve an isometry.

Proposition 1. Let $S$ be any isometry. Let $F=a(\cdot, g) h$ where $\|g\|=$ $\|h\|=1$. Then $S+F$ is isometric if and only if

$$
\left\{\begin{array}{l}
S: g \rightarrow e^{-i \theta} h \\
S^{*}: h \rightarrow e^{\imath \theta} g
\end{array} \text { for some } \theta\right. \text { real }
$$

and

$$
2 \operatorname{Re} e^{-i \theta} a=-|a|^{2} .
$$

Proof. To show $S+F$ is isometric we must show

$$
I=(S+F)^{*}(S+F)=I+S^{*} F+F^{*} S+F^{*} F
$$

or equivalently $S^{*} F+F^{*} S+F^{*} F=0$. Note that $F^{*}=\bar{a}(\cdot, h) g$. If $f \in \mathscr{H}$ then

(a) $S^{*} F f=a e^{i \theta}(f, g) g$,

(b) $F^{*} S f=\bar{a} e^{-i \theta}(f, g) g$, and

(c) $F^{*} F f=|a|^{2}(f, g) g$.

Thus

$$
\left(S^{*} F+F^{*} S+F^{*} F\right) f=\left[\left(a e^{i \theta}+\bar{a} e^{-i \theta}\right)+|a|^{2}\right](f, g) g=0
$$

if (2) holds. 
To prove the necessity assume $S+F$ is isometric whence $S^{*} F+F^{*} S$ $+F^{*} F=0$. The operator $S^{*} F$ is 1 -dimensional and thus in some basis

$$
S^{*} F=\left|\begin{array}{cc:c}
\alpha & 0 & 0 \\
\beta & 0 & 0 \\
\hdashline 0 & 0
\end{array}\right| \text { and } F^{*} S=\left|\begin{array}{cc:c}
\alpha & \beta & 0 \\
0 & 0 & 0 \\
\hdashline 0 & 0 & 0
\end{array}\right|
$$

But $S^{*} F+F^{*} S=-F^{*} F \leq 0$ and it is clear that $S^{*} F+F^{*} S$ cannot be negative unless $\beta=0$. Hence

$$
S^{*} F:\{g\} \rightarrow\{g\} \text { and } S^{*} F:\{g\}^{\perp} \rightarrow 0 .
$$

Set $F=a(\cdot, g) h$ so $F^{*}=\bar{a}(\cdot, h) g$. Since $F^{*} S:\{g\}^{\perp} \rightarrow 0$ if $f \perp g$, then $F^{*} S f=a(S f, h) g=0$, which implies $S f \perp h$. Thus $S:\{g\}^{\perp} \rightarrow\{h\}^{\perp}$ and since $S$ is isometric it follows that $S g=e^{-i \theta} h$ for some $\theta$ real. Hence $S^{*} h=e^{\imath \theta} S^{*} S g=e^{i \theta}$. To see that (2) is necessary we need only repeat the argument from the first half of the proof.

Let $S$ be the unilateral shift on $H^{2}(S: f(z) \rightarrow z f(z))$ and assume $M$ is an invariant subspace for $S$. Then it is well known [2] that $M=\phi H^{2}$ for some inner function $\phi$ in $H^{2}$. Fix $\phi$ for the moment and let $P$ be the self-adjoint projection of $H^{2}$ onto $M^{\perp}$. Let $W f=P S f$ for $f \in M^{\perp}$. It had been observed by Ahern and Clark (see [4]) that there exists a one-dimensional perturbation of $W$ (regarded as an operator on $M^{\perp}$ ) which is unitary. This was pointed out to me by Bernie Morrel. It had also been observed earlier by Ron Douglas (see [3] Corollary 2.3 for example) that one can split off unitary direct summands from the shift by compact perturbations.

Our next result can be said to complement both these results by accomplishing their task in an economical fashion. Thus in Clark's case both the restriction and the perturbation are achieved simultaneously. We denote unitary equivalence by $\cong$.

THEOREM 2. As above, let $S$ denote the unilateral shift on $H^{2}$. For any inner function $\phi$, there exists a one-dimensional operator $F$ such that

$$
S+F \cong U \oplus V \quad \text { on }\left(\phi H^{2}\right)^{\perp} \oplus \phi H^{2}
$$

where $U$ is unitary and $V \cong S$. Moreover, given $\varepsilon>0$, we can choose a $\phi_{\varepsilon}$ such that $S+F_{\varepsilon} \cong U \oplus V$ and $\left\|F_{\varepsilon}\right\|<\varepsilon$.

Proof. Note first that $M=\phi H^{2}$ is spanned by the orthonormal basis $\left\{z^{k} \phi\right\}$ for $k=0,1, \ldots$ Clearly $S \mid M$ is unitarily equivalent to $S$ on $H^{2}$. Set $g=(\overline{\phi(0)} \phi-1) /\|\overline{\phi(0)} \phi-1\|$. Observe that $g \perp \phi$. (If $\phi(0)=0$ then $1 \perp \phi$ which greatly facilitates matters, but leads to an uninteresting situation.) 
We define our one-dimensional operator $F$ as follows:

$$
F=\left(\cdot, S^{*} \varphi\right)(c g-\phi)
$$

where

$$
|c+\phi(0) /\|\bar{\phi}(0) \phi-1\||^{2}=1+|\phi(0)|^{2} /\|\bar{\phi}(0) \phi-1\|^{2} .
$$

Later in the proof we settle on one choice for $c$. However, we need this more general formulation later on.

Several steps are needed to show $F$ has the desired properties.

1. Claim: $S M^{\perp} \subset M^{\perp}+\{\phi\}$. This follows immediately from the observation that $S$ is a contraction and $S^{*}$ is isometric on $M \ominus \phi$. Or note that $S^{*}: M \ominus \phi \rightarrow M$.

(2) If we can show $((S+F) f, \phi)=0$ for $f \in M^{\perp}$ then $S+F$ is clearly $U \oplus V$ on $M^{\perp} \oplus M$, where $U$ is as yet unspecified. But

$$
\begin{aligned}
((S+F) f, \phi) & =(S F, \phi)+\left(f, S^{*} \phi\right)((c g-\phi), \phi) \\
& =(S f, \phi)-\left(f, S^{*} \phi\right)=0 .
\end{aligned}
$$

(3) Claim: $S+F \mid M^{\perp}$ is unitary. To show this we will prove $S+F$ is isometric. The conclusion then follows since

$$
-1=\operatorname{index}(S+F)=\operatorname{index} U+\operatorname{index} V=\operatorname{index} U+(-1)
$$

whence index $U=0$. Hence $U$ must be onto and thus is unitary.

(4) $S+F$ is isometric on $M^{\perp}$. Let $P$ be the self-adjoint projection of $H^{2}$ onto $M \ominus g$. Then for $f \in M^{\perp}$,

$$
\begin{aligned}
\|(S+F) f\|^{2} & =|((S+F) f, g)|^{2}+\|P(S+F) f\|^{2} \\
& =\left|(S f, g)+\left(f, S^{*} \phi\right)(c g-\phi, g)\right|^{2}+\|P S f\|^{2} \\
& =\left|\left(f, S^{*} \phi\right) \phi(0)\|\bar{\phi}(0) \phi-1\|^{-1}+c\left(f, S^{*} \phi\right)\right|^{2}+\|P S f\|^{2} \\
& =\left\|\left.\left(f, S^{*} \phi\right)\right|^{2}\left(1+|\phi(0)|^{2}\|\bar{\phi}(0) \phi-1\|^{-2}\right)+\right\| P S f \|^{2} \\
& =\left.\left\|\left.(S f, \phi)\right|^{2}+\right\|(S f, g)\right|^{2}+\|P S f\|^{2}=\|S f\|^{2}=\|f\|^{2} .
\end{aligned}
$$

(5) Finally we show that we can choose $\phi$ so that the associated $F$ has small norm. One can specify the dimension of $M^{\perp}$ in advance and still achieve this goal as will become obvious in a moment. If $\phi$ is a singular inner function then

$$
\phi(z)=\exp \int \frac{z+e^{i \theta}}{z-e^{i \theta}} d \mu(\theta)
$$

and conversely any singular measure $\mu$ generates such an inner function. It is well known that for such $\phi$ 's, the associated space $\left(\phi H^{2}\right)^{\perp}$ is infinite 
dimensional. Let us consider this case first. Choose $\mu$ such that $\mu(\partial \mathbf{D})=t$. Then $\phi(0)=e^{-t}$. It is at this point, we must exercise some care in the choice of $c$. The most efficient choice (in terms of size) requires that

$$
\arg c=\arg \phi(0)
$$

and

$$
\begin{aligned}
|c| & =\left\{1+|\phi(0)|^{2}\|\bar{\phi}(0) \phi-1\|^{-2}\right\}^{-1 / 2}-|\phi(0)|\|\bar{\phi}(0) \phi-1\|^{-1} \\
& =(1-|\phi(0)|)\left[1-|\phi(0)|^{2}\right]^{-1 / 2}
\end{aligned}
$$

The latter equality follows from the fact that $\|\bar{\phi}(0) \phi-1\|^{2}=\left(1-|\phi(0)|^{2}\right)$. Note that with this choice, $|c| \leq 1$. Hence

$$
\begin{aligned}
\|F\| & \leq\left\|S^{*} \phi\right\|(1+|c|) \leq 2\left[1-|\phi(0)|^{2}\right]^{1 / 2} \\
& =2\left[1-e^{-2 t}\right]^{1 / 2} .
\end{aligned}
$$

Thus for suitably chosen $t$, it follows that $\|F\| \leq \varepsilon$. To handle finite-dimensional $\left(\phi H^{2}\right)^{\perp}$, choose $\phi$ to be a finite Blaschke product where $|\phi(0)|$ is close to 1 .

REMARK. Although one-dimensional perturbations can achieve somewhat surprising results they are limited in certain respects. While it follows from [3] that a compact perturbation of the shift can have the form $S \oplus I$, no one-dimensional perturbation can accomplish this feat as we shall show.

Lemma. Let $T \in \mathscr{L}(\mathscr{H})$ and let $F_{0}$ be an n-dimensional operator. Then

$$
\operatorname{dim} \operatorname{ker}\left(T+F_{0}-\lambda\right) \leq n+\operatorname{dim} \operatorname{ker}(T-\lambda)
$$

for each $\lambda \in \mathbf{C}$.

Proof. Since $F_{0}$ is $n$-dimensional it can be written as $F_{0}=$ $\sum_{j=1}^{n}\left(\cdot, g_{n}\right) h_{n}$. Let $G=\operatorname{clm}\left\{g_{j}: j=1, \ldots, n\right\}$. Fix $\lambda \in \mathbf{C}$ and assume $\operatorname{dim} \operatorname{ker}\left(T+F_{0}-\lambda\right)>n+\operatorname{dim} \operatorname{ker}(T-\lambda)$. It follows from an easy argument that there exists a non-zero vector $f \in \operatorname{ker}\left(T+F_{0}-\lambda\right)$ where $f \perp G$ and $f \perp \operatorname{ker}(T-\lambda)$. Thus

$$
0=\left(T+F_{0}-\lambda\right) f=(T-\lambda) f+\sum\left(f, g_{j}\right) h_{j}=(T-\lambda) f
$$

which is a contradiction.

COROLLARY. There exists no finite dimensional operator $F$ such that

$$
S+F \cong U \oplus V
$$


where $U$ is a unitary operator on an infinite-dimensional space and $\sigma(U)$ is a finte set. In particular $S+F$ cannot be unitarily equivalent to $I \oplus S$.

Proof. Assume such a $U$ exists with $\sigma(U)=\left\{\lambda_{1}, \ldots, \lambda_{k}\right\}$. Then $\operatorname{dim} \operatorname{ker}\left(U-\lambda_{j}\right)$ must be infinite for some $\lambda_{j}$, which contradicts the previous lemma.

EXAMPLE. Since we have shown above that no rank one perturbation of $S$ produces a unitary with singleton spectrum it is interesting to see just what sort of a $U$ is obtained by using the simplest possible singular inner function. So let us take $\phi(z)=e^{(z+1) / z-1)}$. We now compute the spectrum of $U$ by using Theorem 3.2 from [4], assuming we choose $c$ so that $\arg c=\arg \phi(0)$.

Caution: Clark's notation varies from ours. We have used $S$ to denote the unilateral shift while Clark uses $S$ to denote the restriction of the shift to a star invariant subspace. Thus his $S$ and ours do not even act on the same space. Also, because of a slight variation in notation, his $w$ in Theorem 3.2 is equal to $-e^{\arg c}$ when we choose $c$ with $\arg c=\arg \phi(0)$.

Continuing, we see that for $\phi(z)=\exp ((z+1) /(z-1))$ the resulting $U$ has spectrum equal to

$$
\{1\} \cup\{-[1+(2 k+1) \pi i] /[1-(2 k+1) \pi i]: k=0, \pm 1, \ldots\} .
$$

By the lemma above, each spectral point different from 1 is an eigenvalue of multiplicity one. Clark actually exhibits the associated eigenvectors.

COROllary. Let $Q$ be isometric but not purely unitary. Then there exists a one-dimensional operator $F$ such that

$$
Q+F \cong U \oplus Q^{\prime}
$$

where $Q^{\prime}$ is unitarily equivalent to $Q, U$ is unitary and $F$ has arbitrarily small norm.

Proof. Use the Wold decomposition.

2. An application: the Berg process. In Theorem 1 of [1], Berg shows how to pull a unitary piece out of the middle of a unilateral shift while leaving the shift "intact". (Trevor West has christened this process the "Seagull Construction".) Our approach also enables us to do this in a simple geometric fashion. In particular, we require only a rank one perturbation of arbitrarily small norm.

Rather than stating a theorem, we shall simply describe the process and its end result. Let $S$ be our shift on $H^{2}$. Choose a finite Blaschke 
product $\phi$ with $n$ factors. We will later specify $\phi(0)$. We define our first perturbation to be

$$
F_{1}=-1\left(\cdot, S^{*} \phi\right) \phi
$$

Thus $S+F_{1}$ splits as

$$
\frac{\rightarrow}{\left(\phi H^{2}\right)^{\perp}} \frac{\rightarrow}{\phi H^{2}} \quad S+F_{1}
$$

(Also see the matrix at the end of this section.) Of course $S+F_{1}$ is no longer isometric. We select a second Blaschke product $\psi$ of $m$ factors. We set

$$
F_{2}=-1\left(\cdot, S^{*} \phi \psi\right) \phi \psi
$$

Thus $S+F_{1}+F_{2}$ has the representation

$$
\frac{\rightarrow}{\left(\phi H^{2}\right)^{\perp}} \frac{\rightarrow}{\phi H^{2} \ominus \phi \psi H^{2}} \frac{\rightarrow}{\phi \psi H^{2}} \quad S+F_{1}+F_{2}
$$

We define $F_{3}=\left(\cdot, S^{*} \phi\right) \phi \psi$. With this choice of $F_{3}$, it should be clear by construction that $S+F_{1}+F_{2}+F_{3}$ is the unilateral shift on $\left(\phi H^{2}\right)^{\perp}$ $\oplus \phi \psi H^{2}$ and leaves $\phi H^{2} \ominus \phi \psi H^{2}$ invariant as indicated below:

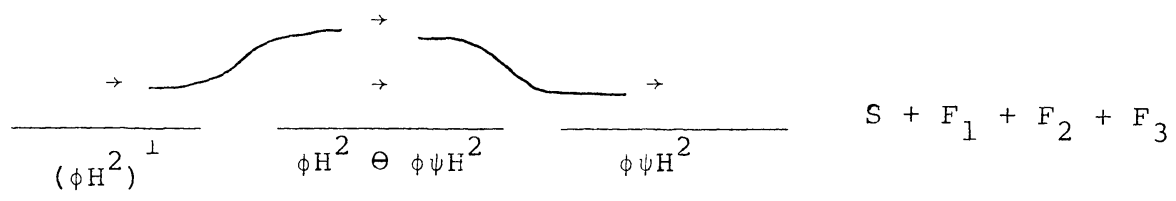

Finally we wish to modify our operator to be unitary on $\phi H^{2} \ominus \phi \psi H^{2}$. To this end define $g=\phi(\bar{\psi}(0) \psi-1) /\|\phi(\bar{\psi}(0) \psi-1)\|$. Note that $g \in$ $\phi H^{2} \ominus \phi \psi H^{2}$ and $\|\phi(\bar{\psi}(0) \psi-1)\|^{2}=1-|\psi(0)|^{2}$.

Set $F_{4}=\left(\cdot, S^{*} \phi \psi\right) c g$ where

( $\alpha) \quad\left|c+\psi(0)\|\phi(\bar{\psi}(0) \psi-1)\|^{-1}\right|^{2}=1+|\psi(0)|^{2}\|\phi(\bar{\psi}(0) \psi-1)\|^{-2}$.

It is easy to see, by repeating the argument in Theorem 2 , that $S+F_{1}+$ $F_{2}+F_{3}+F_{4}$, which is reduced by $\phi H^{2} \ominus \phi \psi H^{2}$, is unitary on that 
subspace if and only if $(\alpha)$ is satisfied. Our operator $S+F_{1}+F_{2}+F_{3}+$ $F_{4}$ can now be represented as

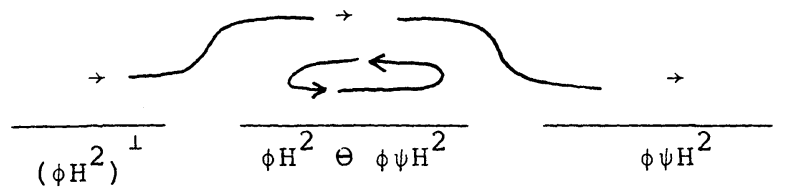

It is this final result which we view as the Berg construction.

However we are far from finished. Certainly $F_{1}+F_{2}+F_{3}+F_{4}$ does not seem to be a rank one operator. Let us group the terms as

$$
F_{1}+F_{3}=\left(\cdot, S^{*} \phi\right)(\phi \psi-\phi)
$$

and

$$
F_{2}+F_{4}=\left(\cdot, S^{*} \phi \psi\right)(c g-\phi \psi) .
$$

Clearly $F=F_{1}+F_{2}+F_{3}+F_{4}$ will be a rank one operator if and only if $(\phi \psi-\phi)$ equals $(c g-\phi \psi)$ modulo the scalars. Performing the obvious arithmetic we see that $F$ is rank one if and only if

$$
c=\|\phi(\bar{\psi}(0) \psi-1)\|(\bar{\psi}(0)-1)^{-1} \text {. }
$$

At this point we would like to satisfy $(\alpha)$ and $(\beta)$ simultaneously. Remarkably enough $(\beta) \Rightarrow(\alpha)$. Indeed if we observe that

$$
\|\phi(\bar{\psi}(0) \psi-1)\|^{2}=\left(1-|\psi(0)|^{2}\right),
$$

then substituting in the left-hand side of $(\alpha)$ we obtain

$$
\begin{aligned}
& \left|\left(1-|\psi(0)|^{2}\right)^{1 / 2}(\bar{\psi}(0)-1)^{-1}+\psi(0)\left(1-|\psi(0)|^{2}\right)^{-1 / 2}\right|^{2} \\
& \quad=\left(1-|\psi(0)|^{2}\right)^{-2}=1+|\psi(0)|^{2}\|\phi(\bar{\psi}(0) \psi-1)\|^{-2} .
\end{aligned}
$$

Thus if we choose $c$ to satisfy $(\beta)$ then our operator $F$ is rank one, and $S+F$ has the required form. To complete matters we need only show that $F$ has any preassigned norm. Note that $\left\|F_{1}\right\|=\left\|S^{*} \phi\right\|=\left(1-|\phi(0)|^{2}\right)^{1 / 2}$. Thus the norm of $F_{1}$ is small for $|\phi(0)|$ close to 1 , and $F_{2}$ and $F_{3}$ can be handled in similar fashion. Since

$$
\left\|F_{4}\right\|=\left\|S^{*} \phi \psi\right\||c|
$$

and $c=\left(1-|\psi(0)|^{2}\right)^{1 / 2}(\bar{\psi}(0)-1)^{-1}$, to handle this case we simply choose $\psi(0)$ to be negative or imaginary and close to 1 in absolute value. Note that $\operatorname{dim}\left(\phi H^{2}\right)^{\perp}=n$ and $\operatorname{dim}\left(\phi H^{2}-\phi \psi H^{2}\right)=m$ so we may specify the size of the pieces if we wish. 


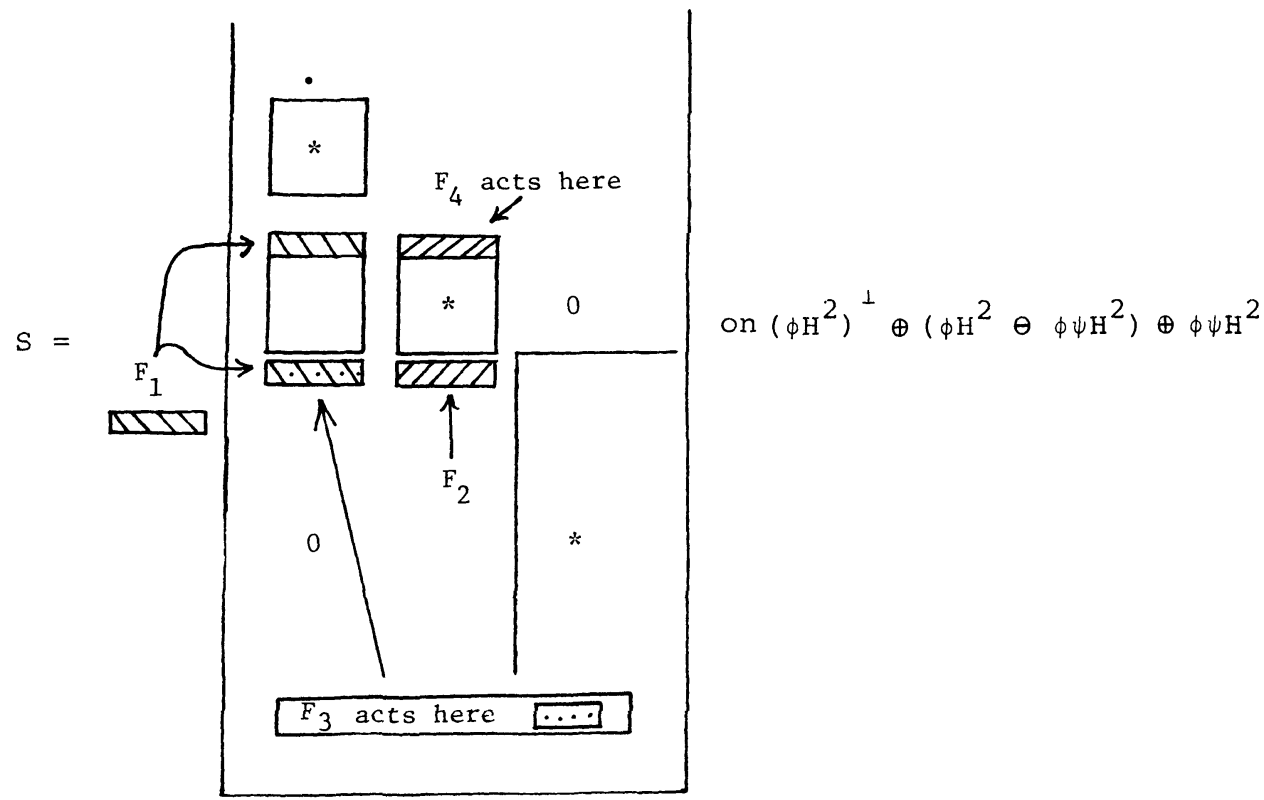

3. In this section we will construct a one-dimensional perturbation of arbitrarily small norm of a diagonal normal operator $D$ which has no eigenvalues. The spectrum of $D$ will be the unit square. This answers a question raised by Hong W. Kim.

Let $\left\{e_{n}\right\}_{n=1}^{\infty}$ be an orthonormal basis for the Hilbert space $\mathscr{H}$. The operator $D$ is defined by

$$
D e_{j}=\lambda_{j} e_{j} \text { for } n=1,2, \ldots
$$

We designate our one-dimensional operator by $F$ where $F f=(f, g) h$ for all $f \in \mathscr{H}$. The vectors $g, h$ can be expressed as $\sum a_{i} e_{i}$ and $\sum b_{i} e_{i}$ respectively. We will now define the $\lambda_{i}$ 's and $b_{i}$ 's in stages.

Consider the unit square $s$ with sides on $\operatorname{Re} z= \pm \frac{1}{2}$ and $\operatorname{Im} z= \pm \frac{1}{2}$. At stage 1 we divide it as follows and assign $\lambda_{1}, \ldots, \lambda_{4}$ as indicated.

\begin{tabular}{|c|c|}
\hline.$\lambda_{1}$ &.$\lambda_{2}$ \\
\hline.$\lambda_{3}$ &.$\lambda_{4}$ \\
\hline
\end{tabular}

At the second step we subdivide each of the boxes in the last subdivision thus obtaining

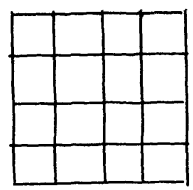


The next $16 \lambda_{i}$ 's are taken to be the midpoints of the indicated squares. We continue in this fashion.

Thus at the $n$th stage we have

(a) $2^{n}$ subintervals per side

(b) length of a subinterval is $2^{-n}$

(c) there are $2^{2 n}$ boxes in the subdivision

(d) we set the next $2^{2 n} b_{i}$ 's equal to $1 / n 2^{n}$ at this stage.

First observe that $h=\sum b_{i} e_{i} \in \mathscr{H}$ since

$$
\|h\|=\sum_{\text {stages vector in } n \text {th stage }} \frac{1}{2^{2 n} n^{2}}=\sum_{\text {stages }} \frac{1}{n^{2}}<\infty .
$$

(The penultimate step follows from the observation that there are $2^{2 n}$ vectors in the $n$th stage.)

We do not define the $a_{i}$ 's for the moment but observe that $a_{i} \neq 0$ for $i=1,2, \ldots$. Assume that $(\lambda-D-F) f=0$ for $\lambda \in S$, the unit square. Then $(\lambda-D) f=(f, g) h$. To rule out the case $(f, g)=0$, observe that it would then follow that $f=e_{j_{0}}$ whence $(f, g)=a_{j_{0}} \neq 0$, a contradiction. Set $f=\sum \mu_{i} e_{i}$. Then $\Sigma\left(\lambda-\lambda_{i}\right) \mu_{i} e_{i}=(f, g) \sum b_{i} e_{i}$ and hence $\mu_{i}=$ $\left(b_{l} /\left(\lambda-\lambda_{i}\right)\right)(f, g)$ for $i=1,2, \ldots$. Without loss of generality we may take $(f, g)=1$. We claim that $f \notin \mathscr{H}$ for $\lambda \in S$. In our computation of the norm of $f$, it makes a slight difference where $\lambda$ is located in $S$. For clarity take $\lambda$ as indicated.

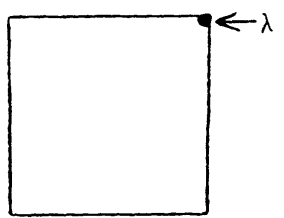

We evaluate $\Sigma\left|b_{j}\right|^{2} /\left|\lambda-\lambda_{j}\right|^{2}$ in stages again. Within a given stage, we group the $\lambda_{j}$ 's into disjoint sets $L_{k}$ as indicated in the picture.

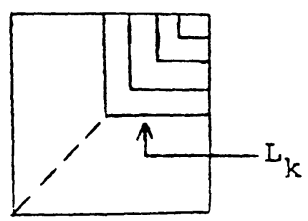

Then we estimate $1 /\left|\lambda-\lambda_{j}\right|$ in each $L$ in the same way. Thus

$$
\begin{aligned}
\|f\|^{2} & =\sum_{j \geq 1}^{\infty} \frac{\left|b_{j}\right|^{2}}{\left|\lambda-\lambda_{j}\right|^{2}}=\sum_{\text {stages vectors in } n \text {th stage }} \frac{\left|b_{j}\right|^{2}}{\left|\lambda-\lambda_{j}\right|^{2}} \\
& \geq \sum_{\text {stages } k=1 \text { vectors in } n \text {th stage }} \sum_{2^{2 n} n^{2}}^{2^{n-1}} 2 k\left(\frac{2^{n}}{k}\right)^{2}
\end{aligned}
$$


(The term $1 / 2^{2 n} n^{2}$ is just $\left|b_{j}\right|^{2}$ and $2^{n} / k$ minimizes the distance from $\lambda$ to $\lambda_{j}$ 's in the $k$ th $L$.)

$$
\begin{aligned}
& \geq \sum_{\text {stages }} n^{-2} \sum_{k=1}^{2^{n-1}} \frac{1}{k} \\
& \geq \sum_{\text {stages }} n^{-2} \log 2^{n-1} \geq \sum \frac{1}{n}=+\infty .
\end{aligned}
$$

Note that this estimate is valid for any placement of $\lambda \in S$. Thus $D+F$ has no eigenvalues for $\lambda \in S$.

To handle the case $\lambda \notin S$ we note that if $(\lambda-D) f=F f$ then $f$ must be a scalar multiple of $(\lambda-D)^{-1} h$. Thus we set $f_{\lambda}=(\lambda-D)^{-1} h=$ $\sum b_{j} e_{j} /\left(\lambda-\lambda_{j}\right)$. Then $\lambda \notin S$ is an eigenvalue of $(D+F)$ if and only if $\left(f_{\lambda}, g\right)=1$. We let $g=\sum a_{i} e_{i}$ where $a_{i}>0$ and choose the $a_{i}$ 's so small that $0<\sum a_{i} b_{i} / \operatorname{Re}\left(1 / 2-\lambda_{i}\right)<1$. But

$$
\operatorname{Re}(f, g)=\sum \operatorname{Re} \frac{a_{i} b_{i}}{\left(\lambda-\lambda_{i}\right)} \leq \sum \frac{a_{i} b_{i}}{\operatorname{Re}\left(1 / 2-\lambda_{i}\right)}<1
$$

for $\operatorname{Re} \lambda \geq \frac{1}{2}$, hence there are no eigenvalues in that half plane.

On the other hand $\operatorname{Re}\left(f_{\lambda}, g\right)<0$ for $\operatorname{Re} \lambda \leq-1 / 2$ so there are no eigenvalues there either.

For $\operatorname{Im} \lambda \geq 1 / 2$ we see that $\operatorname{Im}\left(f_{\lambda}, g\right)>0$ and for $\operatorname{Im} \lambda<-1 / 2$ we have $\operatorname{Im}\left(f_{\lambda}, g\right)<0$ so there are no eigenvalues in those regions. We have exhausted $S^{\prime}$. Thus $D+F$ has no eigenvalues. As constructed,

$$
\|F\|=\|g\|\|h\|=\|g\| \pi / \sqrt{6}=\left[\sum\left|a_{i}\right|^{2}\right]^{1 / 2} \pi / \sqrt{6} .
$$

Thus we may make $\|F\|$ as small as desired by simply choosing the $a_{i}$ 's to be sufficiently small.

REMARK. By a similar construction we can define a self-adjoint diagonal operator $S$ whose spectrum is $[0,1]$, and a one-dimensional self-adjoint operator $F$ such that $S+F$ has no eigenvalues. We omit the details.

\section{REFERENCES}

[1] I. D. Berg, On approximation of normal operators by weighted shifts, Michigan Math. J., 21 (1974), 377-383.

[2] A. Beurling, On two problems concerning linear transformations in Hilbert space, Acta Math., 81 (1949), 239-255. 
[3] L. G. Brown, R. G. Douglas, and P. A. Fillmore, Unitary Equivalence Modulo the Compact Operators and Extension of $C^{*}$-algebras, Lecture Notes in Mathematics, \# 345, Springer-Verlag, New York, 1973.

[4] D. Clark, One-dimensional perturbations of restricted shifts, J. D'Analyse Math., 25 (1972), 169-191.

Received August 31,1981. The author gratefully acknowledges the support of the National Science Foundation.

INDIANA UNIVERSITY

BLOOMINGTON, IN 47405 



\title{
PACIFIC JOURNAL OF MATHEMATICS EDITORS
}

\author{
Donald BABBITt (Managing Editor) \\ University of California \\ Los Angeles, CA 90024 \\ J. DugunduI \\ University of Southern California \\ Los Angeles, CA 90089-1113 \\ R. FINN \\ Stanford University \\ Stanford, CA 94305 \\ HermanN FlaschKa \\ University of Arizona \\ Tucson, AŻ 85721
}

C. C. Moore

University of California

Berkeley, CA 94720

ARTHUR Ogus

University of California

Berkeley, CA 94720

Hugo Rossi

University of Utah

Salt Lake City, UT 84112

H. SAMELSON

Stanford University

Stanford, CA 94305

ASSOCIATE EDITORS
R. ARENS
E. F. BECKENBACH
B. H. NEUMANN
F. WOLF
K. YoSHIDA (1906-1982)

\section{SUPPORTING INSTITUTIONS}

$\begin{array}{ll}\text { UNIVERSITY OF ARIZONA } & \text { UNIVERSITY OF OREGON } \\ \text { UNIVERSITY OF BRITISH COLUMBIA } & \text { UNIVERSITY OF SOUTHERN CALIFORNIA } \\ \text { CALIFORNIA INSTITUTE OF TECHNOLOGY } & \text { STANFORD UNIVERSITY } \\ \text { UNIVERSITY OF CALIFORNIA } & \text { UNIVERSITY OF HAWAII } \\ \text { MONTANA STATE UNIVERSITY } & \text { UNIVERSITY OF TOKYO } \\ \text { UNIVERSITY OF NEVADA, RENO } & \text { UNIVERSITY OF UTAH } \\ \text { NEW MEXICO STATE UNIVERSITY } & \text { WASHINGTON STATE UNIVERSITY } \\ \text { OREGON STATE UNIVERSITY } & \text { UNIVERSITY OF WASHINGTON }\end{array}$

The Supporting Institutions listed above contribute to the cost of publication of this Journal, but they are not owners or publishers and have no responsibility for its content or policies.

Mathematical papers intended for publication in the Pacific Journal of Mathematics should be in typed form or offset-reproduced (not dittoed), double spaced with large margins. Please do not use built up fractions in the text of the manuscript. However, you may use them in the displayed equations. Underline Greek letters in red, German in green, and script in blue. The first paragraph must be capable of being used separately as a synopsis of the entire paper. In particular it should contain no bibliographic references. Please propose a heading for the odd numbered pages of less than 35 characters. Manuscripts, in triplicate, may be sent to any one of the editors. Please classify according to the scheme of Math. Reviews, Index to Vol. 39. Supply name and address of author to whom proofs should be sent. All other communications should be addressed to the managing editor, or Elaine Barth, University of California, Los Angeles, California 90024.

There are page-charges associated with articles appearing in the Pacific Journal of Mathematics. These charges are expected to be paid by the author's University, Government Agency or Company. If the author or authors do not have access to such Institutional support these charges are waived. Single authors will receive 50 free reprints; joint authors will receive a total of 100 free reprints. Additional copies may be obtained at cost in multiples of 50 .

The Pacific Journal of Mathematics is issued monthly as of January 1966. Regular subscription rate: $\$ 190.00$ a year (5 Vols., 10 issues). Special rate: $\$ 66.00$ a year to individual members of supporting institutions.

Subscriptions, orders for numbers issued in the last three calendar years, and changes of address should be sent to Pacific Journal of Mathematics, P.O. Box 969, Carmel Valley, CA 93924, U.S.A. Old back numbers obtainable from Kraus Periodicals Co., Route 100, Millwood, NY 10546.

The Pacific Journal of Mathematics at P.O. Box 969, Carmel Valley, CA 93924 (ISSN 0030-8730) publishes 5 volumes per year. Application to mail at Second-class postage rates is pending at Carmel Valley, California, and additional mailing offices. Postmaster: Send address changes to Pacific Journal of Mathematics, P.O. Box 969, Carmel Valley, CA 93924.

PUBLISHED BY PACIFIC JOURNAL OF MATHEMATICS, A NON-PROFIT CORPORATION

Copyright $@ 1984$ by Pacific Journal of Mathematics 


\section{Pacific Journal of Mathematics}

\section{Vol. 115, No. $2 \quad$ October, 1984}

Ersan Akyildiz, Gysin homomorphism and Schubert calculus ...........257

Marilyn Breen, Clear visibility and unions of two starshaped sets in the

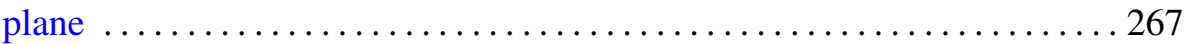

Robert F. Brown, Retraction methods in Nielsen fixed point theory . ......277

Herbert Busemann and Bhalchandra B. Phadke, A general version of Beltrami's theorem in the large ............................... 299

Gerald Arthur Edgar and Robert Francis Wheeler, Topological properties of Banach spaces ............................... 317

Yaakov Friedman and Bernard Russo, Conditional expectation without

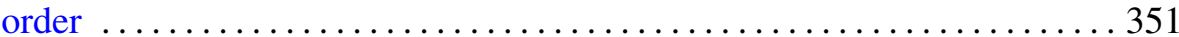

Robert Allen Goggins, Cobordism of manifolds with strong almost tangent

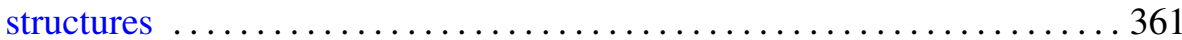

Mike Hoffman, Noncoincidence index of manifolds . . . . . . . . . . . . . 373

William H. Julian, $\varepsilon$-continuity and monotone operations $\ldots \ldots \ldots \ldots 385$

Gerasimos E. Ladas, Y. G. Sficas and I. P. Stavroulakis, Nonoscillatory

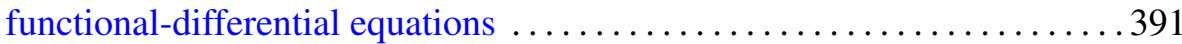

Arnold William Miller and Karel Libor Prikry, When the continuum has

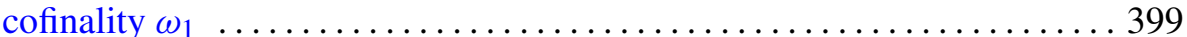

Jean-Leah Mohrherr, Density of a final segment of the truth-table

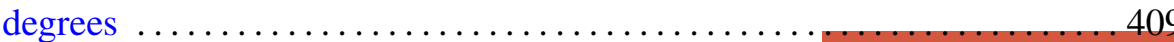

Carl Norman Mutchler, The flat Cauchy problem for radially hyperbolic operators from a characteristic manifold of high codimension ...

Kenji Nakagawa, On the orders of automorphisms of a closed Riemann surface

W. Ricker, Representation of vector-valued functions by Laplace transforms

Jorge Donato Samur, On semigroups of convolution operators in Hilbert

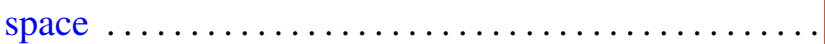

Joseph Gail Stampfli, One-dimensional perturbations of operators 481 Andrew George Earnest and John Sollion Hsia, Correction to: "Spinor norms of local integral rotations. II" 